\title{
Craniotomies following acute traumatic brain injury in Finland-a national study between 1997 and 2018
}

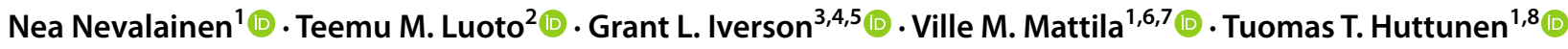

Received: 2 November 2021 / Accepted: 24 January 2022 / Published online: 4 February 2022

(c) The Author(s) 2022

\begin{abstract}
Background A number of patients who sustain a traumatic brain injury (TBI) require surgical intervention due to acute intracranial bleeding. The aim of this retrospective study was to assess the national trends of acute craniotomies following TBI in the Finnish adult population.

Methods The data were collected retrospectively from the Finnish Care Register for Health Care (1997-2018). The study cohort covered all first-time registered craniotomies following TBI in patients aged 18 years or older. A total of 7627 patients (median age $=59$ years, men $=72 \%$ ) were identified.

Results The total annual incidence of acute trauma craniotomies decreased by 33\%, from 8.6/100,000 in 1997 to 5.7/100,000 in 2018. The decrease was seen in both genders and all age groups, as well as all operation subgroups (subdural hematoma, SDH; epidural hematoma, EDH; intracerebral hematoma, ICH). The greatest incidence rate of 15.4/100,000 was found in patients 70 years or older requiring an acute trauma craniotomy. The majority of surgeries were due to an acute SDH and the patients were more often men. The difference between genders decreased with age $(18-39$ years $=84 \%$ men, $40-69=78 \%$ men, $70+$ years $=55 \%$ men). The median age of the patients increased from 58 to 65 years during the 22-year study period. Conclusions The number of trauma craniotomies is gradually decreasing; nonetheless, the incidence of TBI-related craniotomies remains high among geriatric patients. Further studies are needed to determine the indications and derive evidence-based guidelines for the neurosurgical care of older adults with TBIs to meet the challenges of the growing elderly population.
\end{abstract}

Keywords Traumatic brain injury $\cdot$ Neurosurgery $\cdot$ Craniotomy $\cdot$ Epidemiology

This article is part of the Topical Collection on Brain trauma

\section{Nea Nevalainen}

nea.nevalainen@tuni.fi

Teemu M. Luoto

teemu.luoto@pshp.fi

Grant L. Iverson

giverson@mgh.harvard.edu

Ville M. Mattila

ville.mattila@tuni.fi

Tuomas T. Huttunen

tuomas.huttunen@tuni.fi

1 Faculty of Medicine and Health Technology, Tampere University, Tampere, Finland

2 Department of Neurosurgery, Tampere University Hospital and Tampere University, Tampere, Finland
3 Department of Physical Medicine and Rehabilitation, Harvard Medical School, Boston, USA

4 Spaulding Rehabilitation Hospital and Spaulding Research Institute, Boston, USA

5 Home Base, A Red Sox Foundation and Massachusetts General Hospital Program, Charlestown, MA, USA

6 Division of Orthopaedics and Traumatology, Department of Trauma, Musculoskeletal Surgery and Rehabilitation, Tampere University Hospital, Tampere, Finland

7 Coxa Joint Replacement Hospital, Tampere, Finland

8 Department of Cardio-Thoracic Surgery, Tampere Heart Hospital, Tampere University Hospital, Tampere, Finland 


\section{Introduction}

Acute intracranial bleeding can be life-threatening following traumatic brain injury (TBI). A foundation of the emergency management of TBI is to identify the presence of intracranial bleeding and determine whether neurosurgery is required to evacuate blood from the cranial vault. Intracranial abnormalities, visible on day-of-injury computed tomography (CT), are relatively common and occur in a substantial proportion of people presenting to the emergency department (ED) following acute head trauma [49]. The rate of abnormalities increases in association with the severity of injury, as measured by the Glasgow Coma Scale (GCS), whereby those with a GCS score of 15 have fairly low rates $[13,17]$ but those with GCS scores of 14, 13, and lower have much higher rates [15]. The large majority of those cases, however, do not require neurosurgery [12]. The rates of people requiring neurosurgery for an intracranial abnormality, in consecutive series of patients presenting to the ED following head trauma, vary across studies, but are often reported to range between approximately $5 \%$ and $37 \%$ in the group of moderate to severe TBI [9, 21, 25, 33].

People who sustain severe TBIs are at risk for lifelong disability [24]. In Europe, TBI is responsible for the greatest number of total years lived with disability resulting from trauma [24] and it is also one of the injuries with the highest hospital cost per capita [39]. A cost-analysis study in 2018 stated that in Finland the 1-year healthcare cost of all patients with TBI treated in an intensive care unit (ICU) was 122 million USD, and the total costs have increased over the past decade in part due to greater numbers of older adults being admitted to the ICU following injury [41]. As the world's population increases and ages, emergency medicine and neurosurgery will need to adapt and develop the management of TBI in older adults. Increasing frailty and medical co-morbidities place elderly at risk of neurosurgical emergency after injury. Moreover, the use of antithrombotics in the elderly population puts people at risk for intracranial bleeding $[1,32]$.

There are no nationwide studies reporting the incidence of acute neurosurgical management of TBI in the adult population. Better understanding of the incidence rates and temporal trends of trauma-related craniotomies might modify clinical practice and aid health policy-making by informing preventive measures and healthcare resource utilization. The aim of this retrospective cohort study is to assess the national trends of craniotomies following acute TBI in the whole Finnish adult population in 1997-2018. We analyze incidence rates for acute craniotomies following acute subdural hematomas (SDH), epidural hematomas $(\mathrm{EDH})$, and intracerebral hematomas (ICH).
We hypothesized that the incidence of acute craniotomies, especially among the elderly, has increased during the last two decades.

\section{Methods and materials}

Patient data was gathered retrospectively from the Finnish Care Register for Health Care (FCRHC). Patients aged 18 years or older with their date of admission between January 1, 1997 and December 31, 2018 were included. The FCRHC is a nationwide database that includes data on patients discharged from hospital care and contains variables such as service providing unit, age and sex of the patient, duration of hospital stay, all diagnoses (ICD-10, International Classification of Diseases-10 code), external causes and type of accident, and all procedures performed during the hospital stay. Surgical procedures are classified according to NCSP (Nordic Classification of Surgical Procedures) [35]. Both private and public health service providers are obligated to submit information to the FCRHC ensuring excellent national coverage, accuracy, and extent of the database $[16,29,47]$.

The main outcome measure for this population-based study was the annual number and incidence (per 100,000 people per year, hereinafter presented as X/100,000) of hospitalized patients with TBI treated for the first time with an acute neurosurgical operation in Finland. To identify patients treated with acute TBI-related craniotomy, the criteria for selecting the patients were as follows: any of the diagnosis codes were trauma to the head and any of the procedures performed were either evacuation of EDH (NCSP code: AAD00), evacuation of acute SDH (AAD05), or evacuation of traumatic ICH (AAD15). A Finnish version of the NOMESCO (Nordic Medico-Statistical Committee) procedural coding was used [35].

Using the pseudonymized personal identification number, we were able to identify patients hospitalized more than once due to the same procedure and included only the first-time registered hospitalization among the same procedure type. The annual mean population according to age and sex was derived from Statistics Finland Open data pages [36].

Selected data were adjusted by age and sex in order to examine the trend in annual incidence rates of acute TBIrelated neurosurgical operations performed from 1997 to 2018 and to compare incidence rates between subgroups. Incidence rates calculated with the patients gathered from the FCRHC represent the whole adult population (aged 18 years or older) in Finland, and thus statistical estimation methods such as p-values and 95\% confidence intervals were 
not calculated. The data analyses were performed using IBM SPSS Statistics 26.0 (Armonk, NY, USA).

The research protocol for the present study was approved by the research committee of the National Institute of Health and Welfare (Dnro THL/1800/5.05.00/2019). Formal ethical approval or written informed consents were not required, because all the study data were collected retrospectively from national registries, and the data were analyzed in a pseudonymized fashion.

\section{Results}

A total of 7627 patients, 5520 (72\%) men and 2107 (28\%) women, underwent acute craniotomy due to TBI in Finland between 1997 and 2018. Of these, 5235 were for acute SDH evacuation surgeries, 1300 were for traumatic ICH evacuation surgeries, and 1092 underwent surgery for the evacuation of EDH. The median age of the patients was 59 years and during the 22 -year study period the median age increased from 58 years in 1997 to 65 years in 2018. Patients with acute SDH were the oldest (median age 63 years) while patients that underwent EDH surgery were the youngest (median age 43 years). The median age of patients with traumatic ICH was 57 years. Men were younger than women in all procedure groups (Table 1). The median age in men increased from 53 years in 1997 to 62 years in 2018, and in women from 69 years in 1997 to 71 years in 2018 . The trends in median age between 1997 and 2018 among patients who underwent surgery are presented in Fig. 1. The age distribution of the study population is shown in Fig. 2. Over the entire study period, men were over-represented (72.4\%; Fig. 2). The gender difference varied by age $(18-39$ years $=84.5 \%$ men; $40-69$ years $=77.8 \%$ men; and $70+$ years $=55.4 \%$ men; Fig. 2 ).
Table 1 Total number of procedures and median age of surgically treated TBI patients

\begin{tabular}{llllllll}
\hline & & $n$ & $\%$ & $\begin{array}{l}\text { Median age dur- } \\
\text { ing study period }\end{array}$ & $\begin{array}{l}\text { Median age } \\
\text { 1997 (years) }\end{array}$ & $\begin{array}{l}\text { Median age } \\
2018 \text { (years) }\end{array}$ \\
\hline Subdural hematoma & Total & 5235 & - & 63 & 61 & 67 \\
& Men & 3665 & 70 & 60 & 56 & 66 \\
& Women & 1570 & 30 & 70 & 71 & 71 \\
Intracerebral hematoma & Total & 1300 & - & 57 & 61 & 62 \\
& Men & 1007 & 77 & 56 & 54 & 59 \\
& Women & 293 & 23 & 63 & 66 & 72 \\
Epidural hematoma & Total & 1,092 & - & 43 & 44 & 45 \\
& Men & 848 & 78 & 41 & 43 & 41 \\
& Women & 244 & 22 & 52 & 55 & 60 \\
& Total & 7627 & - & 59 & 58 & 65 \\
& Men & 5520 & 72 & 57 & 53 & 62 \\
& Women & 2107 & 28 & 67 & 69 & 71 \\
\hline
\end{tabular}

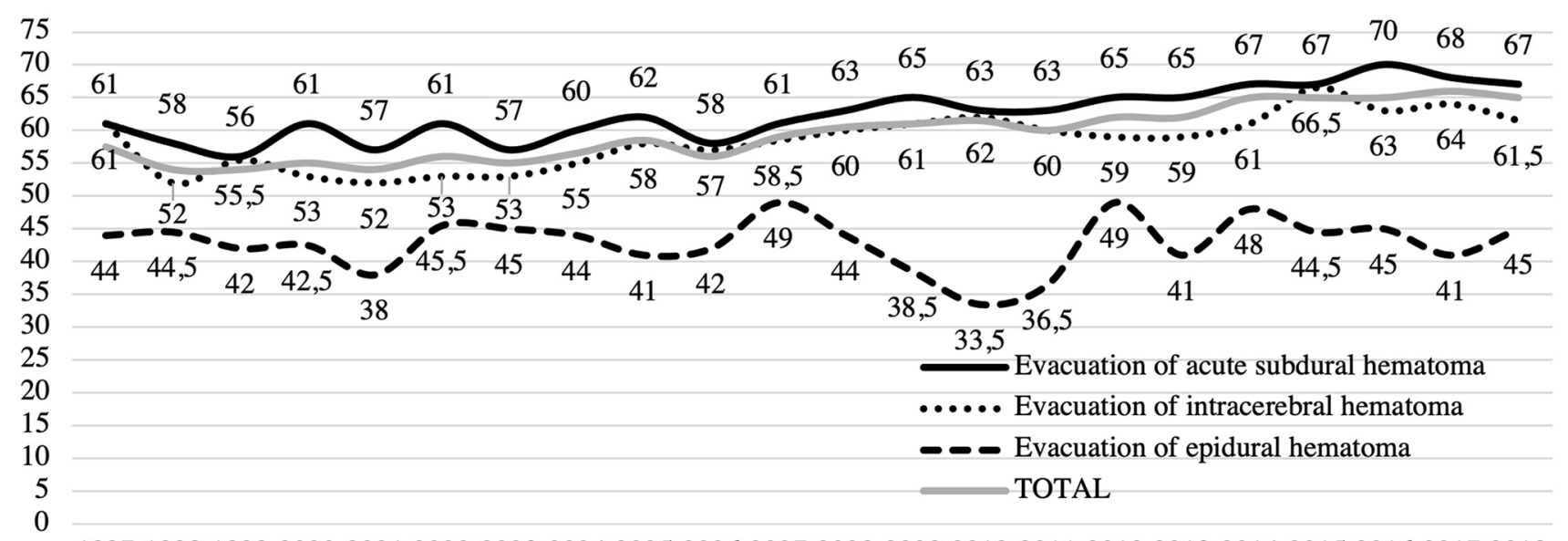

1997199819992000200120022003200420052006200720082009201020112012201320142015201620172018

Fig. 1 Median age of patients who underwent acute craniotomy following TBI between 1997 and 2018 
Fig. 2 Number of patients undergoing neurosurgery between 1997 and 2018 by age and gender

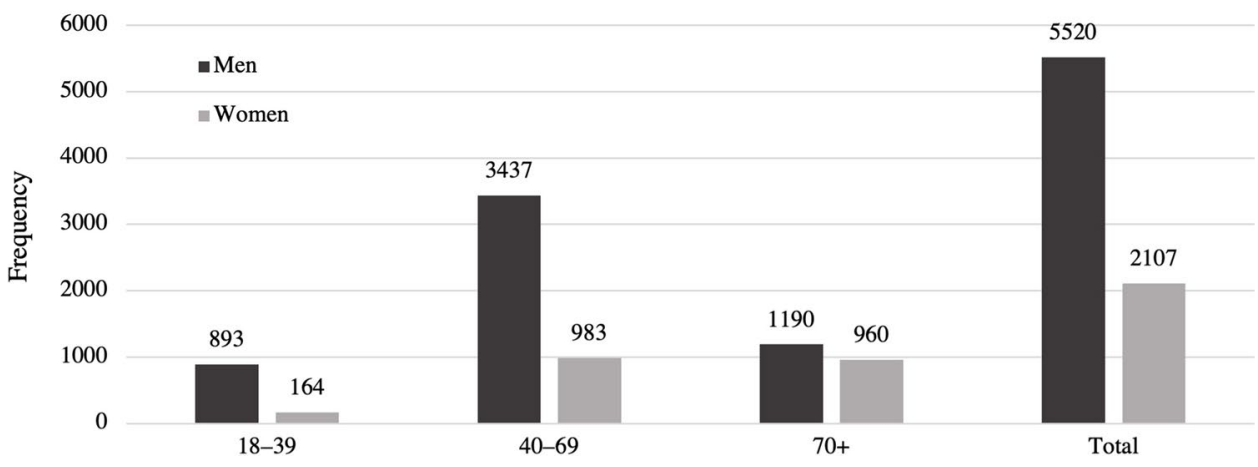

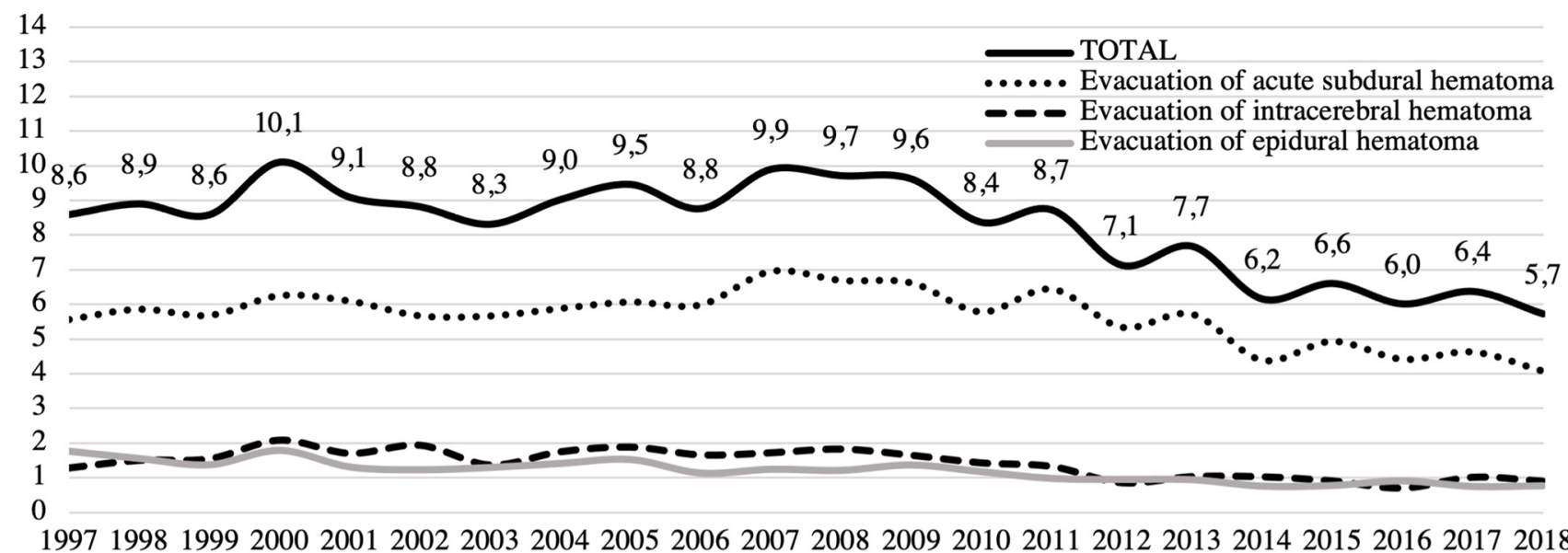

Fig. 3 Incidence (per 100,000) of all acute craniotomies following TBI between 1997 and 2018

Through the study period, the total incidence of acute trauma craniotomies declined by $33 \%$ from 8.6/100,000 in 1997 to $5.7 / 100,000$ in 2018 (Fig. 3). The incidence of acute $\mathrm{SDH}$ evacuation surgeries decreased from 5.6/100,000 in 1997 to 4.1/100,000 in 2018 (decrease of $27 \%$ ). Corresponding numbers for traumatic ICH evacuation surgeries were 1.3/100,000 in 1997 and 0.9/100,000 in 2018 (decrease of $30 \%$ ), and for EDH evacuation surgeries $1.8 / 100,000$ in 1997 and 0.8/100,000 in 2018 (decrease of $57 \%$ ). Men underwent surgery more frequently than women in all procedure groups (Fig. 4).

Evacuation of acute SDH was most common in the elderly population (aged 70 years and older) and least common among young adults (aged 18 to 39 years) between 1997 and 2018. In contrast, the evacuation of EDH was most common among young adults and least common among the elderly. Evacuation of traumatic ICH was as common among elderly as middle-aged patients (aged 40 to 69 years) while it was least common in the group of young adults (Table 2). In total, during the 22-year study period, neurosurgical operations following TBI were most common among patients aged 70 years and older with the incidence rate of 15.4/100,000. A decrease in the incidence of acute craniotomies following TBI was found in all age groups (Fig. 5).

\section{Discussion}

We examined the nationwide trends of TBI-related acute neurosurgical operations in the Finnish adult population over a 22-year period. There were three main findings. First, during the study period, the total incidence rate of emergency craniotomies decreased, in total, by $33 \%$. The decrease was seen in all procedure subgroups as well as in both men and women. Second, men required neurosurgery more frequently than women in all age groups. The male predominance decreased by age as the proportion of men was $85 \%$ between the ages of 18 and 39 years, $78 \%$ between 40 and 69 years, and $55 \%$ in those 70 years and 
older. Finally, the median age of patients increased during the study period (1997: 58 years; and 2018: 65 years).

As expected, acute SDH-related emergency craniotomies were the most common of the procedures and the median age of acute SDH patients was the highest of the three procedure groups (acute SDH, EDH, and ICH). The observed decrease in surgery rates was quite surprising because geriatric TBI in general has been considered to be a growing problem [19]. However, our findings were in line with Flynn-O'Brien and colleagues [10] who reported a decline from 36 to $7 \%$ in surgical intervention of severe TBI between 1995 and 2012. They also reported a likely multifactorial improvement in inpatient mortality in the study population. The contradiction between our findings and the previous reports of increasing geriatric TBI [19] could be explained in part by some reluctance to provide elderly patients with neurosurgery. There is a notable lack of guidelines and prognostic tools for geriatric TBI, thus complicating the neurosurgeon's ability to make evidencebased decisions on surgical treatment. While present treatment guidelines are based on and targeted to younger patients, it is unclear which of the older patients actually benefit from emergency trauma neurosurgery [8, 14]. It is suggested that age alone should not serve as a contraindication for general neurosurgery $[27,50]$. However, surgical decision-making based on local protocols and experience may exclude some elderly patients with TBIs who might benefit from neurosurgery [50]. It is also notable that the elderly patients often present with relatively high GCS scores, although suffering from a significant intracranial hemorrhage [46]. The initial clinical severity stratification of elderly TBI is often underestimated. The justification for performing a trauma craniotomy on a geriatric TBI patient is largely lacking evidence and might also be prone to implicit bias. For example, performing a trauma craniotomy on an 80-year-old patient with a GCS of 14 or 15 and an 11-mm-thick acute SDH is justified according to the current guidelines [4] derived from younger patient cohorts. However, many neurosurgeons would opt not to do surgery on such a case due to a poor long-term prognosis.

Overrepresentation of men was expected. Men are more prone to risk-taking behavior [5]. Men are reported to be predisposed to risk factors such as alcohol intoxication, as well as more likely to encounter situations such as violence and road traffic accidents leading to TBI [22, 28, 37, 44]. Our results align with previous reviews by Brazinova and colleagues [3] and Peeters and colleagues [38]. In both reviews, men are more likely to sustain TBIs than women. A considerable number of TBIs following road traffic accidents and violence incidents occur when the person is under the influence of alcohol [22, 28, 37, 44]. Posti and colleagues [40] reported that the overall incidence rate of fatal TBIs decreased by $4.1 \%$ annually between 2004 and 2016 in Finland. The decrease in TBI mortality was associated with the decrease in overall alcohol consumption. However, this temporal association was not evident in the older TBI population. Additionally, the incidence of road traffic accidents has decreased during our study period (statistics by Finnish Road Safety Council). It is possible that the decreasing rates of neurosurgery for EDH and ICH were related, in part, to societal alcohol consumption and decreasing rates of road traffic accidents. Unfortunately, we were unable to verify this using the present data. In high-income countries, the causes of TBI are gradually changing from high-energy injuries among younger patients to low-energy injuries, such as ground-level falls, among elderly. Older adults are at risk of fall-related TBIs due to frailty, comorbidity, and antithrombotic medication. In high-income countries, increasing lifeexpectancy predisposes older adults to TBI because older individuals stay mobile and live longer with chronic illnesses $[19,43]$. To further prevent the increase of elderly falls and therefore TBI, prevention methods such as strength and balance training, use of vitamin D and calcium supplements, and professional home-hazard assessment is pointed to be effective [20].

Over the two-decade-long study period, the median age of patients who underwent acute TBI craniotomies increased by 7 years (from 58 to 65). The median age increased in all operation types. The most notable increase was found among male acute SDH patients whose median age increased by 10 years (from 56 to 66 years) during the study period. We assume the increase in the median age is due, in part, to the growing elderly population. Moreover, it can be speculated that in recent years neurosurgery is offered more frequently to elderly TBI patients compared to the end of the twentieth century. A growing body of evidence suggests that chronological age and TBI severity alone are insufficient to accurately predict outcome in older adults who sustain TBIs [6, 23, 26, 48]. Historically, elderly patients with mass lesions were considered to be cases with extremely poor outcomes regardless of treatment intensity. Thus, these patients with an assumed poor prognosis have been treated conservatively, simultaneously generating a self-fulfilling prophecy [31, 42]. Undoubtedly, some surgical treatment bias and nihilism toward elderly patients with TBIs still exists as pointed out earlier [45]. Moreover, the use of antithrombotic medication can contribute to the changing trends in the age distribution of trauma craniotomies [11]. The use of antithrombotics is increasing especially among individuals aged 65 years or older [1]. Approximately $30 \%$ of patients aged 65 years or older admitted to Level 1 trauma centers after a low-level fall and $33 \%$ of head trauma patients aged 55 years or older are on pre-injury antithrombotics [32, 34]. However, acute reversal strategies are available for some but not all oral anticoagulation drugs as well as antiplatelet agents [7, 51]. 
a

10.0

9.0

8.0

7.0

6.0

5.0

4.0

3.0

2.0

1.0

0.0

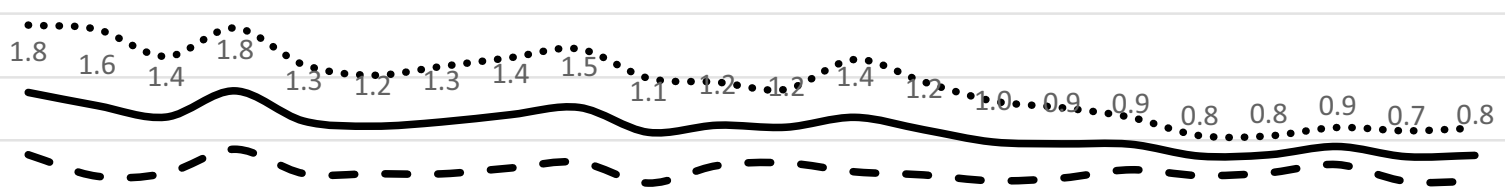

1997199819992000200120022003200420052006200720082009201020112012201320142015201620172018

b

10.0

9.0

8.0

7.0

6.0

5.0

4.0

3.0

2.0

1.0

0.0

1997199819992000200120022003200420052006200720082009201020112012201320142015201620172018

c

10.0

9.0

8.0

7.0

6.0

5.0

4.0

3.0

2.0

1.0

0.0

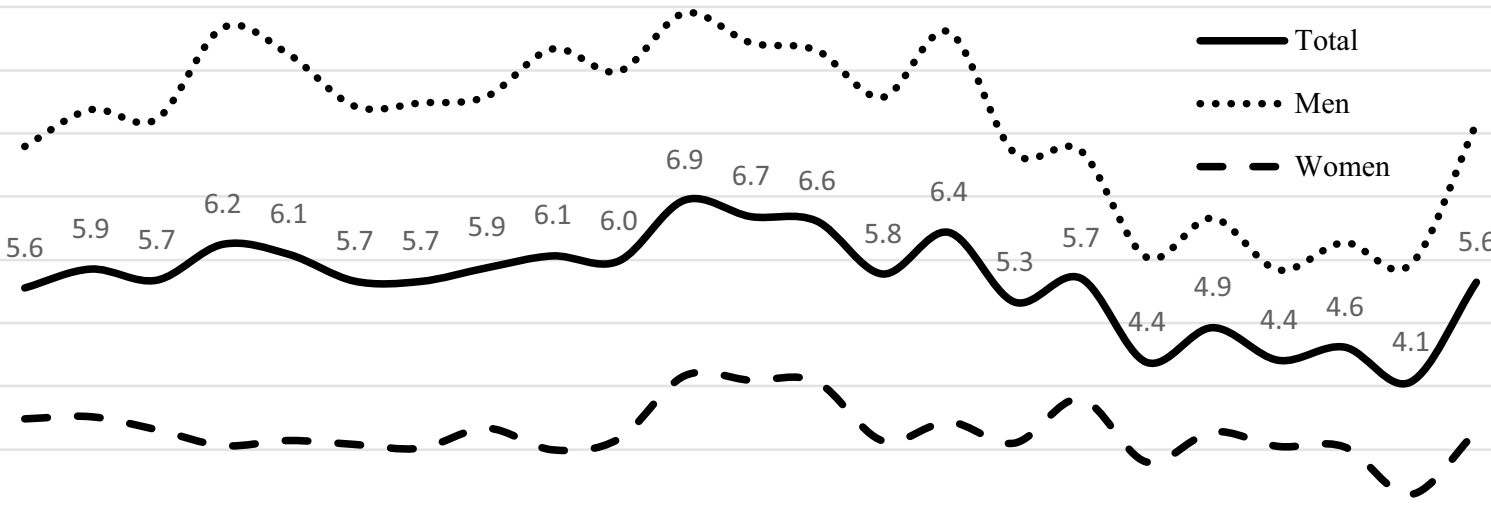


4Fig. 4 Incidence (per 100,000) of men and women who underwent EDH surgery (a), SDH surgery (b), and ICH surgery (c) between 1997 and 2018 in Finland

Table 2 Incidence of procedures presented by sex and age group in the Finnish adult population in 1997-2018

\begin{tabular}{llll}
\hline Incidence/100,000 $(n)$ & \multicolumn{3}{l}{ Age (years) } \\
\cline { 2 - 4 } & $18-39$ & $40-69$ & $70+$ \\
\hline Subdural Hematoma & $1.3(434)$ & $6.5(3000)$ & $12.9(1801)$ \\
Men & $2.1(356)$ & $10.1(2317)$ & $18.5(992)$ \\
Women & $0.5(78)$ & $2.9(683)$ & $9.4(809)$ \\
Intracerebral hematoma & $0.5(151)$ & $1.9(888)$ & $1.9(261)$ \\
Men & $0.8(132)$ & $3.1(714)$ & $3.0(161)$ \\
Women & $0.1(19)$ & $0.7(174)$ & $1.2(100)$ \\
Epidural hematoma & $1.4(472)$ & $1.2(532)$ & $0.6(88)$ \\
Men & $2.4(405)$ & $1.8(406)$ & $0.7(37)$ \\
Women & $0.4(67)$ & $0.5(126)$ & $0.6(51)$ \\
Total & $3.2(1057)$ & $9.6(4420)$ & $15.4(2150)$ \\
Men & $5.3(893)$ & $15.0(3437)$ & $22.2(1190)$ \\
Women & $1.0(164)$ & $4.2(983)$ & $11.1(960)$ \\
\hline
\end{tabular}

Prothrombin complex concentrate (PCC) therapy is shown to be effective in the rapid lowering of INR in the case of traumatic coagulopathy in patients with or without the pre-injury use of warfarin $[2,18]$. The use of PCC therapy can quickly facilitate neurosurgical care of anticoagulated patients with ICH [2]. It is also shown that treatment with recombinant activated factor VII rapidly after the onset of $\mathrm{ICH}$ can reduce the growth of the hematoma [30]. It is uncertain whether the growth of the hematoma can be sufficiently reduced for conservative treatment to be used instead of surgical evacuation of the hematoma.

The strength of our study is the high quality of the FCRHC database for epidemiologic research. The data collection for FCRHC is mandatory for both public and private health care services and institutions in Finland and therefore has excellent nationwide coverage and accuracy [16, 29, 47]. Our strengths also include a long study period of 22 years and that the Finnish healthcare system is based on public healthcare entitled equally to everyone residing in Finland ensuring that the data reflects the actual annual need of emergency craniotomies.

The weakness of our study is that the FCRHC does not provide information about whether the patients hospitalized several times during the 22 years of follow-up had been admitted again due to the same TBI, revision of the previous operation, or due to a new TBI. Therefore, we included only the first hospitalization of each patient, hence every patient is represented only once in our data. Therefore, it is possible that we slightly underestimated the incidence of craniotomies following TBIs. Another weakness of the FCRHC is the lack of data about TBI severity, other treatments or outcomes, the patient's comorbidities, or other risk factors such as medications or alcohol consumption that increase the risk of TBI. Furthermore, decompressive craniectomy procedures (code: AAK80) were excluded from the study due to NCSP coding inconsistencies. The AAK80 code was only introduced in 2007. During our study period, a total of 304 first-time registered decompressive craniectomies following TBI were reported in the database, with an annual incidence of 0.1-0.9 per 100,000 between 2008 and 2018. Because decompressive craniectomies were not included, the true incidence of acute surgical procedures following TBI is likely slightly higher.

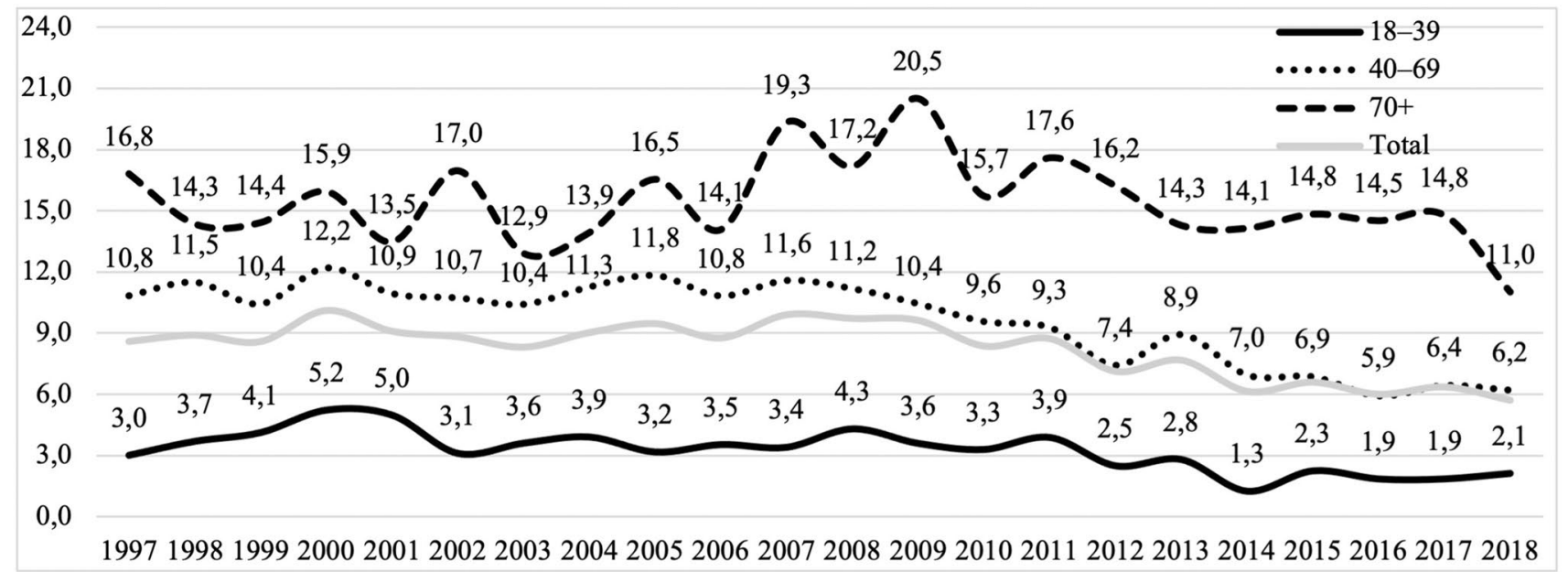

Fig. 5 Incidence (per 100,000) of all acute craniotomies following TBI between 1997 and 2018 stratified by age groups 
In conclusion, the incidence of acute craniotomies following TBI has gradually decreased during the last two decades. Simultaneously, the age of neurosurgically treated patients with TBIs has increased. The majority of patients requiring trauma neurosurgery are men and require surgery due an acute SDH. Further studies are needed to determine the indications and derive evidence-based guidelines for the neurosurgical care of older adults with TBIs to meet the challenges of the growing elderly population.

Acknowledgements The authors have no acknowledgements to address.

Author contribution Study design: TML and TTH; statistical analyses: NERN and TTH; first manuscript draft: NERN; manuscript editing: NERN, TML, TTH, VMM, and GLI; and pre-submission manuscript approval: NERN, TML, TTH, VMM, and GLI.

\section{Funding Not applicable.}

Data availability The original pseudonymized data is not freely available according to the GDPR regulations.

Code availability The code and analyses used are available per request.

\section{Declarations}

Ethics approval The research protocol for the present study was approved by the research committee of the National Institute of Health and Welfare (Dnro THL/1800/5.05.00/2019). Formal ethical approval or written informed consents were not required, because all the study data were collected retrospectively from national registries, and the data were analyzed in a pseudonymized fashion.

Conflict of interest Grant Iverson serves as a scientific advisor for NanoDX® (formerly BioDirection, Inc.), Sway Operations, LLC, and Highmark, Inc. He has a clinical and consulting practice in forensic neuropsychology, including expert testimony, involving individuals who have sustained mild TBIs. He has received research funding from the National Football League. He has also received research funding from the Harvard Integrated Program to Protect and Improve the Health of National Football League Players Association Members. He has received unrestricted philanthropic support from ImPACT Applications, Inc., the Mooney-Reed Charitable Foundation, Boston Bolts, Heinz Family Foundation, National Rugby League, and the Spaulding Research Institute. Nea Nevalainen, Teemu Luoto, Ville Mattila, and Tuomas Huttunen report no competing financial interests.

Open Access This article is licensed under a Creative Commons Attribution 4.0 International License, which permits use, sharing, adaptation, distribution and reproduction in any medium or format, as long as you give appropriate credit to the original author(s) and the source, provide a link to the Creative Commons licence, and indicate if changes were made. The images or other third party material in this article are included in the article's Creative Commons licence, unless indicated otherwise in a credit line to the material. If material is not included in the article's Creative Commons licence and your intended use is not permitted by statutory regulation or exceeds the permitted use, you will need to obtain permission directly from the copyright holder. To view a copy of this licence, visit http://creativecommons.org/licenses/by/4.0/.

\section{References}

1. Adelborg K, Grove EL, Sundbøll J, Laursen M, Schmidt M (2016) Sixteen-year nationwide trends in antithrombotic drug use in Denmark and its correlation with landmark studies. Heart 102(23):1883-1889

2. Beynon C, Nofal M, Rizos T, Laible M, Sakowitz OW, Unterberg AW (2020) Prothrombin complex concentrate for vitamin K antagonist reversal in traumatic intracranial hemorrhage. J Clin Neurosci Off J Neurosurg Soc Australas 79:197-202

3. Brazinova A, Rehorcikova V, Taylor MS et al (2018) Epidemiology of traumatic brain injury in Europe: a living systematic review. J Neurotrauma. https://doi.org/10.1089/neu.2015.4126

4. Bullock MR, Chesnut R, Ghajar J, Gordon D, Hartl R, Newell DW, Servadei F, Walters BC, Wilberger JE (2006) Surgical management of acute subdural hematomas. Neurosurgery 58(3 Suppl):S16-24; discussion Si-iv

5. Byrnes JP, Miller DC, Schafer WD (1999) Gender differences in risk taking: a meta-analysis. Psychol Bull 125:367-383

6. De Bonis P, Pompucci A, Mangiola A, D’Alessandris QG, Rigante L, Anile C (2010) Decompressive craniectomy for the treatment of traumatic brain injury: does an age limit exist? A review J Neurosurg 112(5):1150-1153

7. Elgamal H, Luedi MM, Ender JK, Meineri M (2020) Preoperative management of anticoagulation in the surgical patient: Highlights of the latest guidelines. Best Pract Res Clin Anaesthesiol 34(2):141-152

8. Evans LR, Jones J, Lee HQ, Gantner D, Jaison A, Matthew J, Fitzgerald MC, Rosenfeld JV, Hunn MK, Tee JW (2019) Prognosis of acute subdural hematoma in the elderly: a systematic review. J Neurotrauma 36(4):517-522

9. Firsching R, Woischneck D (2001) Present status of neurosurgical trauma in Germany. World J Surg 25(9):1221-1223

10. Flynn-O'Brien KT, Fawcett VJ, Nixon ZA et al (2015) Temporal trends in surgical intervention for severe traumatic brain injury caused by extra-axial hemorrhage, 1995 to 2012. Neurosurg 76(4):451-460

11. Gaist D, García Rodríguez LA, Hellfritzsch M, Poulsen FR, Halle B, Hallas J, Pottegård A (2017) Association of antithrombotic drug use with subdural hematoma risk. JAMA 317(8):836-846

12. Galgano M, Toshkezi G, Qiu X, Russell T, Chin L, Zhao LR (2017) Traumatic brain injury: current treatment strategies and future endeavors. Cell Transplant 26(7):1118-1130

13. Ganti L, Stead T, Daneshvar Y, Bodhit AN, Pulvino C, Ayala SW, Peters KR (2019) GCS 15: when mild TBI isn't so mild. Neurol Res Pract 1:6

14. Gardner RC, Dams-O'Connor K, Morrissey MR, Manley GT (2018) Geriatric traumatic brain injury: Epidemiology, outcomes, knowledge gaps, and future directions. J Neurotrauma 35(7):889-906

15. Heskestad B, Baardsen R, Helseth E, Romner B, Waterloo K, Ingebrigtsen $\mathrm{T}$ (2009) Incidence of hospital referred head injuries in Norway: a population based survey from the Stavanger region. Scand J Trauma Resusc Emerg Med 17:6

16. Huttunen TT, Kannus P, Pihlajamäki H, Mattila VM (2014) Pertrochanteric fracture of the femur in the Finnish National Hospital Discharge Register: validity of procedural coding, external cause for injury and diagnosis. BMC Musculoskelet Disord 15:98

17. Isokuortti H, Iverson GL, Silverberg ND, Kataja A, Brander A, Öhman J, Luoto TM (2018) Characterizing the type and location of intracranial abnormalities in mild traumatic brain injury. $\mathrm{J}$ Neurosurg 129(6): 1588-1597

18. Joseph B, Amini A, Friese RS, Houdek M, Hays D, Kulvatunyou N, Wynne J, O'Keeffe T, Latifi R, Rhee P (2012) Factor IX 
complex for the correction of traumatic coagulopathy. J Trauma Acute Care Surg 72(4):828-834

19. Kannus P, Niemi S, Parkkari J, Mattila V, Sievänen H (2020) Fallinduced hospital-treated traumatic brain injuries among elderly Finns in 1970-2017. Arch Gerontol Geriatr 86:103958

20. Kannus P, Sievänen H, Palvanen M, Järvinen T, Parkkari J (2005) Prevention of falls and consequent injuries in elderly people. Lancet (London, England) 366(9500):1885-1893

21. Kay A, Teasdale G (2001) Head injury in the United Kingdom. World J Surg 25(9):1210-1220

22. Korcha RA, Cherpitel CJ, Witbrodt J, Borges G, Hejazi-Bazargan S, Bond JC, Ye Y, Gmel G (2014) Violence-related injury and gender: the role of alcohol and alcohol combined with illicit drugs. Drug Alcohol Rev 33(1):43-50

23. Lilley EJ, Williams KJ, Schneider EB, Hammouda K, Salim A, Haider AH, Cooper Z (2016) Intensity of treatment, end-of-life care, and mortality for older patients with severe traumatic brain injury. J Trauma Acute Care Surg 80(6):998-1004

24. Maas AI, Stocchetti N, Bullock R (2008) Moderate and severe traumatic brain injury in adults. Lancet Neurol 7(8):728-741

25. Maegele M, Lefering R, Sakowitz O, Kopp MA, Schwab JM, Steudel W-I, Unterberg A, Hoffmann R, Uhl E, Marzi I (2019) The incidence and management of moderate to severe head injury. Dtsch Arztebl Int 116(10):167-173

26. Mak CHK, Wong SKH, Wong GK, Ng S, Wang KKW, Lam PK, Poon WS (2012) Traumatic brain injury in the elderly: is it as bad as we think? Curr Transl Geriatr Exp Gerontol Rep 1(3):171-178

27. Maldaner N, Sarnthein J, Bozinov O, Regli L, Neidert MC (2018) Neurosurgery in Octogenarians: A Prospective Study of Perioperative Morbidity, Mortality, and Complications in Elderly Patients. World Neurosurg 110:e287-e295

28. Mäntykoski T, Iverson GL, Renko J, Kataja A, Öhman J, Luoto TM (2019) Violence-related traumatic brain injury. Brain Inj 33(8):1045-1049

29. Mattila VM, Sillanpää P, Iivonen T, Parkkari J, Kannus P, Pihlajamäki H (2008) Coverage and accuracy of diagnosis of cruciate ligament injury in the Finnish National Hospital Discharge Register. Injury 39(12):1373-1376

30. Mayer SA, Brun NC, Begtrup K, Broderick J, Davis S, Diringer MN, Skolnick BE, Steiner T (2005) Recombinant activated factor VII for acute intracerebral hemorrhage. N Engl J Med 352(8):777-785

31. McCredie VA, Alali AS, Xiong W, Rubenfeld GD, Cuthbertson BH, Scales DC, Nathens AB (2016) Timing of withdrawal of lifesustaining therapies in severe traumatic brain injury: Impact on overall mortality. J Trauma Acute Care Surg 80(3):484-491

32. Meade MJ, Tumati A, Chantachote C, Huang EC, Rutigliano DN, Rubano JA, Vosswinkel JA, Jawa RS (2021) Antithrombotic agent use in elderly patients sustaining low-level falls. J Surg Res 258:216-223

33. Murray GD, Teasdale GM, Braakman R et al (1999) The European Brain Injury Consortium survey of head injuries. Acta Neurochir (Wien) 141(3):223-236

34. Narum S, Brørs O, Stokland O, Kringen MK (2016) Mortality among head trauma patients taking preinjury antithrombotic agents: A retrospective cohort analysis from a Level 1 trauma centre. BMC Emerg Med 16(1):29

35. NOMESCO Classification of Surgical Procedures (NCSP) [referred: 9.12.2020]. Access method: https://norden.diva-portal. org/
36. Official Statistics of Finland (OSF): Population structure [e-publication]. ISSN=1797-5395. Helsinki: Statistics Finland [referred: 11.10.2020]. Access method: http://www.stat.fi/til/vaerak/index_ en.html

37. Orsi C, Ferraro OE, Montomoli C, Otte D, Morandi A (2014) Alcohol consumption, helmet use and head trauma in cycling collisions in Germany. Accid Anal Prev 65:97-104

38. Peeters W, van den Brande R, Polinder S, Brazinova A, Steyerberg EW, Lingsma HF, Maas AIR (2015) Epidemiology of traumatic brain injury in Europe. Acta Neurochir (Wien) 157(10):1683-1696

39. Polinder S, Meerding WJ, Van Baar ME et al (2005) Cost estimation of injury-related hospital admissions in 10 European countries. J Trauma - Inj Infect Crit Care 59(6):1283-1290

40. Posti JP, Sankinen M, Sipilä JOT, Ruuskanen JO, Rinne J, Rautava P, Kytö V (2019) Fatal traumatic brain injuries during 13 years of successive alcohol tax increases in Finland - a nationwide population-based registry study. Sci Rep 9(1):5419

41. Raj R, Bendel S, Reinikainen M et al (2018) Temporal trends in healthcare costs and outcome following ICU admission after traumatic brain injury. Crit Care Med 46(4):e302-e309

42. Robertsen A, Førde R, Skaga NO, Helseth E (2017) Treatmentlimiting decisions in patients with severe traumatic brain injury in a Norwegian regional trauma center. Scand J Trauma Resusc Emerg Med 25(1):44

43. Roozenbeek B, Maas AIR, Menon DK (2013) Changing patterns in the epidemiology of traumatic brain injury. Nat Rev Neurol 9(4):231-236

44. Savola O, Niemelä O, Hillbom M (2005) Alcohol intake and the pattern of trauma in young adults and working aged people admitted after trauma. Alcohol Alcohol 40(4):269-273

45. Skaansar O, Tverdal C, Rønning PA, Skogen K, Brommeland T, Røise O, Aarhus M, Andelic N, Helseth E (2020) Traumatic brain injury-the effects of patient age on treatment intensity and mortality. BMC Neurol 20(1):376

46. Stocchetti N, Carbonara M, Citerio G, Ercole A, Skrifvars MB, Smielewski P, Zoerle T, Menon DK (2017) Severe traumatic brain injury: targeted management in the intensive care unit. Lancet Neurol 16(6):452-464

47. Sund R (2012) Quality of the Finnish Hospital Discharge Register: A systematic review. Scand J Public Health 40(6):505-515

48. Taussky P, Hidalgo ET, Landolt H, Fandino J (2012) Age and salvageability: analysis of outcome of patients older than 65 years undergoing craniotomy for acute traumatic subdural hematoma. World Neurosurg 78(3-4):306-311

49. Tverdal C, Aarhus M, Andelic N, Skaansar O, Skogen K, Helseth E (2020) Characteristics of traumatic brain injury patients with abnormal neuroimaging in Southeast Norway. Inj Epidemiol $7(1): 45$

50. Whitehouse KJ, Jeyaretna DS, Wright A, Whitfield PC (2016) Neurosurgical care in the elderly: increasing demands necessitate future healthcare planning. World Neurosurg 87:446-454

51. Yee J, Kaide CG (2019) Emergency Reversal of Anticoagulation. West J Emerg Med 20(5):770-783

Publisher's note Springer Nature remains neutral with regard to jurisdictional claims in published maps and institutional affiliations. 